\title{
Exploring the Crystal Structure and Functional Role of the Lectin Domain from the Staphylococcal Biofilm Protein Aap
}

Joseph J. Maciag ${ }^{1}$, Catherine T. Chaton ${ }^{1,2}$, Alexander Yarawsky ${ }^{1}$ and Andrew B. Herr ${ }^{1}$

${ }^{1}$ Divisions of Immunobiology and Infectious Diseases, Cincinnati Children's Hospital Medical Center, Cincinnati, OH 45229

${ }^{2}$ Department of Molecular \& Cellular Biochemistry and Center for Structural Biology, University of Kentucky, Lexington, KY 40536, USA

Email Contact: joseph.maciag@cchmc.org

Staphylococcus epidermidis is commensal gram-positive bacterium that colonizes human epithelium. Although commonly viewed as a non-infectious skin microbe, S. epidermidis has been implicated as a major causative agent in hospital-acquired infections, specifically due to its ability to form biofilms on catheters and implanted medical devices. Biofilms are surface-adherent bacterial colonies that are highly resistant to antibiotic action or host immune responses. S. epidermidis utilizes the multifunctional accumulation associated protein (Aap) to facilitate both adhesion to host cell surfaces, mediating initial colonization, and intercellular adherence between staphylococci, leading to biofilm formation. Aap is expressed as an extracellular, fibrillar protein consisting of multiple domains: an Arepeat region, a globular lectin domain, tandem B-repeats, a proline/glycine-rich stalk, and an LPXTG cell wall-anchoring motif. The B-repeat region responsible for intercellular adhesion in the biofilm has been studied extensively, whereas almost nothing is known about the Aap lectin domain. Here, we

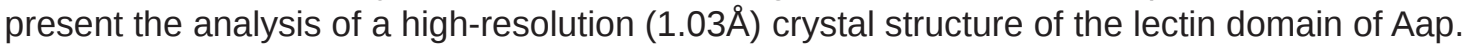

Experimental phases were determined by S-SAD, measuring anomalous scattering of two native sulfur atoms and an intrinsically bound calcium ion. Although the fold has structural similarity to other known lectin family members, the putative binding site differs from other calcium dependent lectins. Through the use of analytical ultracentrifugation (AUC) we were able demonstrate that Aap lectin sediments as a single monomeric species in solution. Additionally, we have analyzed the overall conformation of an N-terminal construct of Aap, in solution, through the implementation of small angle $x$-ray scattering (SAXS). We are continuing to explore the glycan specificity of the lectin domain using high-throughput glycan array screening. These studies of the lectin domain will lay the framework for the design of inhibitors of staphylococcal colonization by blocking their ability to directly interact with their binding targets on human epidermal cells. 